\title{
Radiographie des réseaux parfaits
}

\author{
Christian Batut et Jacques Martinet
}

TABLE DES MATIÈRES

1. Introduction

2. Les réseaux parfaits

3. Inclusions entre réseaux

4. Sections de petite codimension

Appendice : Quelques réseaux classiques

Références
Recherche effectuée au sein de l'unité mixte C.N.R.S. Enseignement Supérieur U.R.M. 9936

Keywords: perfect quadratic forms, perfect lattices
Soit $\Lambda$ un réseau d'un espace euclidien de dimension $n$, contenant $n$ vecteurs minimaux indépendants (une condition satisfaite en particulier par les réseaux parfaits au sens de Voronoï). Nous étudions dans cet article la perfection des réseaux relatifs engendrés par des vecteurs minimaux de $\Lambda$ dans le cas de certains réseaux «classiques », le plus souvent contenus dans le réseau de Leech.

Let $\Lambda$ be a lattice in a Euclidean space of dimension $n$, containing $n$ independant minimal vectors (this condition is verified in particular by perfect lattices in the sense of Voronoi). We study in this article the property of perfection for relative lattices spanned by minimal vectors of $\Lambda$ for some "classical" lattices $\Lambda$, for the most part contained in the Leech lattice.

\section{INTRODUCTION}

La notion de réseau parfait, ou plus classiquement de forme quadratique parfaite (voir définitions ci-dessous), a fait l'objet de nombreux travaux depuis qu'elle a été considérée (sans qu'ils ne lui donnent de nom) par Korkine et Zolotareff. Plus près de nous, Conway et Sloane [1988b] ont fait un bilan de nos connaissances sur les réseaux parfaits dans le cas des petites dimensions, et notre article est en quelque sorte une suite à cet ouvrage.

La notion de perfection ne fait intervenir que l'ensemble des vecteurs minimaux des réseaux. En outre, l'étude de la perfection d'un réseau $\Lambda$ donné est facilitée lorsque $\Lambda$ possède des sections parfaites de même norme de petite codimension ; en particulier, dans le cas de la codimension 1, la perfection est simplement l'existence de $n$ vecteurs minimaux indépendants en-dehors de la section considérée.

Pour ces raisons, il est intéressant de savoir pour un réseau donné, supposé le plus souvent parfait, quelles sont ses sections parfaites de même norme. 
C'est cette question que nous étudions dans cet article.

Après un $\S 2$ consacré à des rappels sur la perfection, nous étudions au $\S 3$ la possibilité de plonger un réseau donné dans un réseau de dimension supérieure en respectant les normes minimales, étude précisée dans le cas où le réseau que l'on plonge est l'un des 48 (à isométrie près) réseaux parfaits de dimension $\leq 7$. Le $\S 4$ est consacré au plongement des réseaux de petite codimension, et plus particulièrement au cas des sections hyperplanes. L'article s'achève par un appendice contenant des rappels sur quelques réseaux classiques.

\section{LES RÉSEAUX PARFAITS}

Soit $E$ un espace vectoriel euclidien de dimension $n$. On appelle norme d'un vecteur $x \in E$, et l'on note $N(x)$, le carré scalaire $x . x$ de $x$ (donc, $N(x)$ est le carré de la norme euclidienne $\|x\|$ de $x$ ).

Soit $\Lambda$ un réseau de $E$. La norme (minimale) de $\Lambda$ est

$$
N=N(\Lambda)=\min _{\substack{x \in \Lambda \\ x \neq 0}} N(x) .
$$

On note $S(\Lambda)$ l'ensemble des vecteurs minimaux de $\Lambda$ (c'est-à-dire des vecteurs de norme $N(\Lambda)$ ), et $D=\operatorname{det} \Lambda$ le déterminant de $\Lambda$. Rappelons que, étant donnée une base $\mathcal{B}=\left(e_{1} \ldots, e_{n}\right)$ de $\Lambda$, on appelle $A=\left(e_{i} . e_{j}\right)$ la matrice de Gram de $\mathcal{B}$; on a $\operatorname{det} \Lambda=\operatorname{det} A$, et l'on dit que $\Lambda$ est entier si les coefficients d'une de ses matrices de Gram sont entiers. Cette condition équivaut à l'inclusion $\Lambda \subset$ $\Lambda^{*}$, où $\Lambda^{*}$ désigne le réseau dual de $\Lambda$. On pose

$$
s=s(\Lambda)=\frac{1}{2}|S(\Lambda)|
$$

c'est la moitié du nombre de contacts («kissing number ») de $\Lambda$.

Les réseaux qui interviennent dans cet article sont tous proportionnels à des réseaux entiers, et donc à un unique réseau $\Lambda$ primitif, c'est-à-dire tel que les produits scalaires deux à deux de vecteurs du réseau engendrent $\mathbb{Z}$. La norme minimale et le déterminant de ce réseau primitif sont alors des invariants de similitude.

Un autre invariant de similitude, qui joue un rôle important dans la suite, est l'invariant de Smith, suite $\left(q_{1}, q_{2}, \ldots, q_{n}\right)$ des diviseurs élémentaires du couple $\left(\Lambda^{*}, \Lambda\right)$, définie ainsi : on a

$$
\Lambda^{*} / \Lambda \simeq \mathbb{Z} / q_{1} \mathbb{Z} \oplus \mathbb{Z} / q_{2} \mathbb{Z} \oplus \cdots \oplus \mathbb{Z} / q_{n} \mathbb{Z}
$$

et $q_{i}$ divise $q_{i-1}$ pour $2 \leq i \leq n$. On notera $q$ le premier terme $q_{1}$ de l'invariant de Smith; c'est l'annulateur du $\mathbb{Z}$-module $\Lambda^{*} / \Lambda$.

$\mathrm{Vu}$ le caractère primitif de $\Lambda$, on a $q_{n}=1$ et l'invariant de Smith de $\Lambda^{*}$ est la suite $\left(q_{i}^{*}=q_{1} / q_{n-i}\right)$, ce que se démontre en observant que $\sqrt{q} \Lambda^{*}$ est le réseau entier et primitif proportionnel à $\Lambda^{*}$.

On utilisera généralement la notation simplifiée $\operatorname{Smith}(\Lambda)=q_{1} \cdot q_{2} \ldots q_{n}$ (à ne pas confondre avec le produit des $q_{i}$, qui est égal au déterminant de $\Lambda$ ), et le plus souvent sous une forme abrégée obtenue en supprimant les facteurs égaux à 1 et en écrivant par exemple $12^{2} \cdot 6.2^{3}$ plutôt que 12.12.6.2.2.2.

De la même façon que l'invariant de Smith permet de préciser l'information donnée par le déterminant, la notion de spectre permet de compléter l'information fournie par la donnée des invariants $N$ et $s$. Le spectre de $x \in S(\Lambda)$ est la suite des entiers $n_{0}, \ldots, n_{k}$, où $k=[N / 2]$ et $n_{i}$ désigne le nombre de couples $\pm y$ de vecteurs minimaux autres que $\pm x$ tels que $|x . y|=i$ [Conway and Sloane 1988 b, p. 57]. Le spectre (minimal) de $\Lambda$ est l'ensemble des suites $n_{0}, \ldots, n_{k}$, représentant le spectre d'un vecteur de $S(\Lambda)$, chaque suite étant affectée d'un coefficient égal au nombre de vecteurs minimaux ayant cette suite pour spectre.

(Plus généralement, il est indispensable - par exemple, pour reconnaître des réseaux ou pour calculer des groupes d'automorphismes - de considérer la notion de spectre obtenue en remplaçant $S(\Lambda)$ par l'ensemble $S_{K}(\Lambda)$ des vecteurs de $\Lambda$ de norme $\leq K$, où $K$ est un réel assez grand pour que $S_{K}(\Lambda)$ contienne une base de $\Lambda$. Toutefois, le choix $K=N$ est suffisant dans les applications aux réseaux qui interviennent dans cet article.) 
Rappelons la définition d'un réseau parfait, notion due à Korkine et Zolotareff et étudiée en détail par Voronoï dans le langage des formes quadratiques: dans le langage géométrique de [Bergé et Martinet 1989], il s'agit d'un réseau tel que l'espace End $^{s} E$ des endomorphismes symétriques de $E$ est engendré par les projections orthogonales $p_{x}$ sur les vecteurs $x \in S(\Lambda)$.

Si l'on associe à un réseau $\Lambda$ muni d'une $\mathbb{Z}$-base $\mathcal{B}$ la forme quadratique ${ }^{t} X A X$, où $A$ est la matrice de Gram de $\mathcal{B}$, et si l'on exprime la matrice de $p_{x}$ dans les bases $\mathcal{B}^{*}$ et $\mathcal{B}$, on retrouve la définition de la perfection dans le langage des formes quadratiques définies positives: $Q={ }^{t} X A X$ est parfaite si les matrices $X$ et ${ }^{t} X$ engendrent l'espace $\operatorname{Sym}_{n}$ des matrices carrées d'ordre $n$ lorsque $X$ parcourt l'ensemble $S(Q) \subset \mathbb{Z}^{n}$ des vecteurs minimaux de $Q$. C'est à l'aide de cette propriété que se teste numériquement la perfection, qui se ramène à un calcul de rang dans $\mathbb{R}^{n(n+1) / 2}$, et en fait dans $\mathbb{Z}^{n(n+1) / 2}$ (voir ci-dessous).

On notera qu'un réseau est parfait si et seulement si son sous-réseau engendré par ses vecteurs minimaux est lui-même parfait. (Il s'agit bien d'un sous-réseau, car on vérifie sans peine que les vecteurs minimaux d'un réseau parfait engendrent $E$.) Korkine et Zolotareff [1877] ont démontré que les réseaux parfaits sont proportionnels à des réseaux entiers, et Voronoï [1908] a prouvé que, dans une dimension donnée, le nombre de classes de similitude de réseaux parfaits est fini.

La classification des réseaux parfaits a été faite jusqu'à la dimension 7 . Nous utilisons les notations de [Conway et Sloane 1988b]. Les 48 classes de réseaux parfaits de dimension $n \leq 7$ sont notées $P_{n}^{i}$, l'indice $i$ variant de 1 à $n_{i}$, avec $n_{1}=n_{2}=n_{3}=1$, $n_{4}=2, n_{5}=3, n_{6}=7, n_{7}=33$. (Pour $n=7$, nous avons remplacé $p_{7}^{i}$ par $P_{7}^{i}$, Jaquet [1993] ayant prouvé que la liste des 33 réseaux parfaits trouvés par Kaye Stacey est effectivement complète). Les réseaux $P_{n}^{i}$ sont définis dans [Conway et Sloane 1988b] par une matrice de Gram à coefficients entiers et premiers entre eux dans leur ensemble. La norme du réseau est le plus petit entier figurant sur la diagonale. Pour chaque dimension $n$, les ensembles des normes qui interviennent sont les suivants :

\begin{tabular}{|l|l|}
\hline \multicolumn{1}{|c|}{$n$} & \multicolumn{1}{c|}{ normes } \\
\hline 1 & 1 \\
$2,3,4$ & 2 \\
5,6 & 2,4 \\
7 & $2,3,4,6,8,10$ \\
\hline
\end{tabular}

En dimension 8, la classsification est loin d'être complète. En utilisant une généralisation de l'algorithme de Voronoï, Laïhem [1992] a construit tous les réseaux parfaits de dimension 8 ayant une section parfaite de même norme de dimension 7 , pourvu que celle-ci ne soit pas l'un des trois réseaux de racines $E_{7}=P_{7}^{1}, D_{7}=P_{7}^{4}, A_{7}=P_{7}^{33}$. Il y a 1171 réseaux parfaits de ce type, notés $L h_{i}$ pour $1 \leq i \leq 1171$ et rangés par densité décroissante (au sens large). Seuls quatre réseaux ont été trouvés au-dessus de l'un des réseaux de racines $E_{7}, D_{7}$ et $A_{7}$, à savoir, $E_{8}$, le réseau $A_{8}^{2}$ de Barnes [1959], $D_{8}$ et $A_{8}$. Cette liste est vraisemblablement complète.

On ne connaît que quelques réseaux parfaits dépourvus de section parfaite de dimension 7 de même norme (Jaquet, Baril, Napias), et l'on n'a aucune idée de leur nombre.

\section{INCLUSIONS ENTRE RÉSEAUX}

On se donne un réseau $\Lambda$ entier dans l'espace $E$ de dimension $n$, pour lequel on conserve les notations du paragraphe précédent; en particulier, on pose $N=N(\Lambda), D=\operatorname{det} \Lambda$ et $s=s(\Lambda)$, et l'on note $q$ l'annulateur de $\Lambda / \Lambda^{*}$, plus petit entier tel que $\Lambda^{\prime}=\sqrt{q} \Lambda^{*}$ soit entier. On note $N^{*}, D^{*}=D^{-1}$ et $s^{*}$ les invariants analogues de $\Lambda^{*}$. Le réseau $\Lambda^{\prime}$ est entier, de déterminant $D^{\prime}=q^{n} D^{-1}$ et de norme $N^{\prime}=q N^{*}$.

On se donne par ailleurs un réseau $M$ également entier, de dimension $m \leq n$ et de déterminant $d$, et l'on cherche si le réseau déduit de $M$ par renormalisation à la norme de $\Lambda$ se plonge dans $\Lambda$. 
Une condition nécessaire pour qu'il en soit ainsi est que la norme de $M$ divise celle de $N$. Cette remarque s'applique au réseau $P_{7}^{2} \sim E_{7}^{*}$, qui est entier de norme 3 , et ne contient donc pas de section parfaite de même norme et de dimension $>1$, puisque les réseaux parfaits entiers et primitifs de dimensions comprises entre 2 et 6 ont pour norme minimale 2 ou 4.

Nous supposons dans la suite que la norme de $M$ divise celle de $\Lambda$, et nous nous ramenons alors par homothétie au cas où $M$ a en fait pour norme $N=N(\Lambda)$. Nous supposons en outre que $M$ n'est pas de dimension 1, cas dans lequel le plongement de $M$ dans $\Lambda$ est trivialement possible.

Supposons $M$ plongé dans $\Lambda$. Soit $F$ le sousespace $\mathbb{R} M$ de $E$. L'intersection $F \cap \Lambda$ est un réseau de $F$ contenant $M$ comme sous-groupe d'indice fini. On dira qu'un tel réseau est une section de $\Lambda$.

L'application qui à une section $L$ de $\Lambda$ associe son orthogonal $L^{\perp}$ dans $\Lambda^{*}$ met en bijection les sections de dimension $m$ de $\Lambda$ et les sections de dimension $n-m$ de $\Lambda^{*}$. Le déterminant de $L^{\perp}$ se calcule tout de suite au moyen de la relation

$$
\operatorname{det} L=\operatorname{det} \Lambda \operatorname{det} L^{\perp} .
$$

Au prix d'une renormalisation, on peut remplacer $\Lambda^{*}$ par $\Lambda^{\prime}$, ce qui a pour effet de multiplier le déterminant de $\Lambda^{\perp}$ par $q^{n-m}$. Revenant au réseau $M$, on constate que la relation précédente prend alors la forme

$$
\frac{\operatorname{det} M}{[\mathbb{R} M: M]^{2}}=\operatorname{det} \Lambda q^{-(n-m)} \operatorname{det} M_{\Lambda^{\prime}}^{\perp} .
$$

Comme le produit $[\mathbb{R} M: M]^{2} \operatorname{det} M_{\Lambda^{\prime}}^{\perp}$ est entier, nous avons démontré:

Théorème 3.1. Si un réseau $M$ entier ayant même norme que $\Lambda$ se plonge dans $\Lambda$, alors le déterminant de $\Lambda$ divise le produit $q^{n-m} \operatorname{det} M$.

Dans les exemples qui suivent, on renvoie à l'appendice et à [Conway et Sloane 1988b] pour les données numériques utilisées.
Exemple 3.2. Dans le cas du réseau $\Lambda=K_{12}$, on a $D=3^{6}$ et $q=3$. Il en résulte qu'un réseau $M$ entier de norme 2 ou 4 et de dimension $\geq 7$ ne peut pas se plonger dans $K_{12}$ si son déterminant n'est pas divisible par 3. Cela s'applique en particulier aux réseaux $E_{7}, D_{7}$ et $A_{7}$ dont les déterminants sont des puissances de 2 .

$\mathrm{Vu}$ l'inclusion $A_{7} \subset A_{8}$, on ne peut pas non plus plonger $A_{8}$ dans $K_{12}$, un résultat pour lequel l'argument précédent ne s'applique pas, puisque $A_{8}$ est de déterminant 9 .

Exemple 3.3. De même, comme $\operatorname{Smith}\left(P_{6}^{5}\right)=7^{3}$ et que les réseaux parfaits de dimension inférieure ont tous des déterminants premiers à 7 , le réseau $P_{6}^{5}$ ne possède pas de section parfaite de même norme dans les dimensions 4 et 5 . En revanche, on vérifie facilement que $A_{2}$ et $A_{3}$ se plongent dans $P_{6}^{5}$.

Exemple 3.4. Dans le cas de $\Lambda=P_{5}^{2}$, on a $D=2.3^{4}$ et $q=6$, ce qui prouve que $P_{5}^{2}$ n'a pas de section parfaite de même norme dans les dimensions 3 et 4. Dans le cas de $P_{6}^{2} \sim E_{6}^{*}$, les égalités $D=3^{5}$ et $q=3$ prouvent que $P_{6}^{2}$ n'a pas d'autres sections parfaites de même norme que $P_{2}^{1}$ et $P_{5}^{2}$, lesquelles sont effectivement des sections. Un argument analogue montre que les sections parfaites de même norme des réseaux $P_{6}^{4}$ et $P_{6}^{6}$ sont $P_{2}^{1}, P_{3}^{1}$ et $P_{5}^{2}$.

La considération des spectres des réseaux $M$ et $\Lambda$ permet aussi de prouver des impossibilités. En effet, si le plongement $M \subset \Lambda$ est possible, pour tout $x \in S(M)$, le nombre de vecteurs de $S(M)$ ayant avec $x$ un produit scalaire donné est majoré par le nombre correspondant de vecteurs de $S(\Lambda)$. On en déduit une relation d'ordre sur les spectres des vecteurs qui se prolonge aux spectres eux-mêmes, et le spectre de $\Lambda$ doit être supérieur à celui de $M$.

Un cas particulier souvent utile concerne les produits scalaires nuls: si les produits scalaires mutuels de $S(\Lambda)$ ne prennent pas la valeur 0 , il doit en être de même de ceux de $M$.

Exemple 3.5. Les réseaux de racines de dimension au moins 3 possèdent des couples de vecteurs minimaux orthogonaux, alors que les réseaux $P_{5}^{3}$ et 
$P_{6}^{2}$ n'en possèdent pas. On retrouve ainsi les résultats sur ces réseaux donnés dans l'exemple 3.4.

Exemple 3.6. Le réseau $P_{7}^{12}$ ne possède pas non plus de couple de vecteurs minimaux ortogonaux. $\mathrm{Vu}$ que sa norme est 6 , ses sections parfaites maximales de norme 6 ne peuvent être que des réseaux semblables à $A_{2}$, lesquels sont effectivement contenus dans $P_{7}^{12}$.

Nous avons écrit un programme permettant de tester l'existence d'une inclusion $M \subset \Lambda$ pour deux réseaux entiers $M$ et $\Lambda$ donnés. Le programme renormalise $M$ à la norme de $\Lambda$ et prend en compte les impossibilités éventuelles liées aux valeurs des normes et du déterminant et à la compatibilité des spectres. Lorsqu'il ne détecte pas d'impossibilité a priori, il cherche à appliquer une base donnée $\left(e_{1}, \ldots, e_{m}\right)$ de $M$ sur une famille $\left(f_{1}, \ldots, f_{m}\right)$ de vecteurs minimaux de $\Lambda$ de mêmes spectres, en utilisant un algorithme de backtracking analogue à celui qui est largement utilisé dans la recherche d'une isométrie entre deux réseaux donnés.

Les 47 réseaux parfaits de dimension comprise entre 2 et 7 étant connus, la recherche de sections parfaites de dimension $\leq 7$ d'un réseau $\Lambda$ donné peut alors se faire par voie informatique. Le programme recherche les inclusions des réseaux $P_{m}^{i}$ dans $\Lambda$ pour $m$ croissant de 2 à $\min (n-1,7)$, et exploite la liste des inclusions entre les réseaux $P_{m}^{i}$ pour éliminer ceux de ces réseaux qui contiennent un réseau dont l'impossibilité du plongement a été démontrée auparavant. On s'arrête à $m=n-1$ parce que le cas $m=n$ est facile à résoudre: l'indice d'un sous-réseau $M$ d'un réseau $\Lambda$ est égal à la racine du quotient des déterminants. Pour des réseaux parfaits de dimension $\leq 7$ et de même norme, ce quotient est majoré par 4 , et l'égalité n'a lieu que dans le cas $M=P_{7}^{33} \simeq A_{7}, \Lambda=P_{7}^{1} \simeq E_{7}$. On peut effectivement plonger $A_{7}$ dans $E_{7}$; c'est l'identification $E_{7}=A_{7}^{2}$ de Coxeter [1951].

Le programme a d'abord été appliqué aux 46 réseaux parfaits de dimensions comprises entre 3 et 7, et a établi la liste de toutes les inclusions $P_{m}^{i} \subset$ $P_{n}^{j}$ avec $m<n$. Le tableau 1 montre les résultats.

\begin{tabular}{|c|ccc|ccccc|}
\hline & \multicolumn{1}{|c|}{ aleurs de $i$ pour $m=$} \\
$(n, j)$ & Smith & $N^{\prime}$ & $s^{*}$ & 2 & 3 & 4 & 5 & 6 \\
\hline$(3,1)$ & 4 & 3 & 4 & 1 & - & - & - & - \\
\hline$(4,1)$ & $2^{2}$ & 2 & 12 & 1 & 1 & - & - & - \\
$(4,2)$ & 5 & 4 & 5 & 1 & 1 & - & - & - \\
\hline$(5,1)$ & 4 & 4 & 5 & 1 & 1 & 1,2 & - & - \\
$(5,2)$ & $6.3^{2}$ & 3 & 10 & 1 & & & - & - \\
$(5,3)$ & 6 & 5 & 6 & 1 & 1 & 2 & - & - \\
\hline$(6,1)$ & 3 & 4 & 27 & 1 & 1 & 1,2 & 1,3 & - \\
$(6,2)$ & $3^{5}$ & 2 & 36 & 1 & & & 2 & - \\
$(6,3)$ & $2^{2}$ & 2 & 6 & 1 & 1 & 1,2 & 1,3 & - \\
$(6,4)$ & 18.6 .3 & 9 & 2 & 1 & 1 & & 2 & - \\
$(6,5)$ & $7^{3}$ & 4 & 21 & 1 & 1 & & & - \\
$(6,6)$ & $39.3^{2}$ & 9 & 2 & 1 & 1 & & 2 & - \\
$(6,7)$ & 7 & 6 & 7 & 1 & 1 & 2 & 3 & - \\
\hline$(7,1)$ & 2 & 3 & 28 & 1 & 1 & 1,2 & 1,3 & $1,3,7$ \\
$(7,2)$ & $2^{6}$ & 2 & 63 & & & & & \\
$(7,3)$ & $18.3^{3}$ & 9 & 1 & 1 & 1 & & 2 & $2,4,6$ \\
$(7,4)$ & 4 & 4 & 7 & 1 & 1 & 1,2 & 1,3 & 3,7 \\
$(7,5)$ & $32.4^{2}$ & 16 & 1 & 1 & 1 & 1,2 & 1 & \\
$(7,6)$ & $48.12 .4 .2^{2}$ & 16 & 1 & 1 & 1 & & & \\
$(7,7)$ & 180.3 & 100 & 3 & 1 & 1 & 1,2 & 2 & 4,6 \\
$(7,8)$ & 48.6 .2 & 27 & 4 & 1 & 1 & 1,2 & 2 & 4 \\
$(7,9)$ & $7280.7^{2}$ & 1568 & 2 & 1 & 1 & & & \\
$(7,10)$ & 84.7 & 48 & 4 & 1 & 1 & 1,2 & & 5 \\
$(7,11)$ & $2520.2^{2}$ & 963 & 4 & 1 & 1 & 1,2 & & \\
$(7,12)$ & $40.4^{4}$ & 15 & 6 & 1 & & & & \\
$(7,13)$ & 2480.31 & 725 & 5 & 1 & 1 & & & \\
$(7,14)$ & $2584.2^{2}$ & 912 & 1 & 1 & 1 & & & \\
$(7,15)$ & 394.197 & 115 & 7 & 1 & 1 & & & \\
$(7,16)$ & $72.18 .2^{3}$ & 27 & 4 & 1 & 1 & 1 & & \\
$(7,17)$ & $2632.2^{2}$ & 988 & 2 & 1 & 1 & & & \\
$(7,18)$ & $19720.2^{2}$ & 5675 & 2 & 1 & 1 & & & \\
$(7,19)$ & 168.21 .3 & 64 & 6 & 1 & 1 & 2 & & \\
$(7,20)$ & 146.73 & 59 & 9 & 1 & 1 & & & \\
$(7,21)$ & 336.16 .2 & 124 & 4 & 1 & 1 & 2 & & \\
$(7,22)$ & 210.3 & 108 & 2 & 1 & 1 & & 2 & 4,6 \\
$(7,23)$ & 1540.7 & 560 & 1 & 1 & 1 & & & \\
$(7,24)$ & 10808 & 3920 & 1 & 1 & 1 & & & \\
$(7,25)$ & $688.4^{2}$ & 248 & 1 & 1 & 1 & & & \\
$(7,26)$ & $18.6^{2}$ & 9 & 3 & 1 & 1 & 2 & 2 & 4 \\
$(7,27)$ & $24.3^{3}$ & 13 & 8 & 1 & 1 & & 2 & 4 \\
$(7,28)$ & 216.3 & 112 & 3 & 1 & 1 & 1,2 & 2 & 6 \\
$(7,29)$ & 228.3 & 108 & 1 & 1 & 1 & 2 & 2 & 4,6 \\
$(7,30)$ & 98.7 & 49 & 1 & 1 & 1 & & & 5 \\
$(7,31)$ & 240.3 & 117 & 2 & 1 & 1 & 2 & 2 & 6 \\
$(7,32)$ & $84.3^{2}$ & 39 & 2 & 1 & 1 & 2 & 2 & 6 \\
$(7,33)$ & 8 & 7 & 8 & 1 & 1 & 2 & 3 & 7 \\
\hline
\end{tabular}

TABLEAU 1. Pour chaque réseau parfait $P_{n}^{j}$ avec $n \leq 7$, on donne les invariants Smith, $N^{\prime}$ et $s^{*}$. En plus on donne, pour $m<n$, les indices $i$ tels que $P_{m}^{i}$ apparaisse comme section minimale de $P_{n}^{j}$ après renormalisation à la même norme minimale. 


\begin{tabular}{|c|c|c|c|c|c|c|}
\hline \multirow[b]{2}{*}{$\Lambda$} & \multicolumn{6}{|c|}{ valeurs de $j$ pour $m=$} \\
\hline & 2 & 3 & 4 & 5 & 6 & 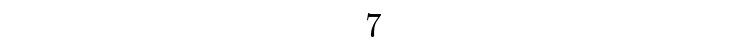 \\
\hline$K_{7}$ & 1 & 1 & 1,2 & 1,3 & 1,3 & - \\
\hline$K_{8}$ & 1 & 1 & 1,2 & 1,3 & 1,3 & \\
\hline$K_{8}^{\prime}$ & 1 & 1 & & 2 & $2,4,6$ & 3,27 \\
\hline$\Lambda_{8}$ & 1 & 1 & 1,2 & 1,3 & $1,3,7$ & $1,4,33$ \\
\hline$A_{8}^{(2)}$ & 1 & 1 & 1,2 & 2 & $2,4,6$ & $3,7,28$ \\
\hline$K_{9}$ & 1 & 1 & 1,2 & $1,2,3$ & $1,3,4,5,6,7$ & $7,10,26,27,28,32$ \\
\hline$K_{9}^{\prime}$ & 1 & 1 & 1,2 & 2,3 & $2,4,6$ & $3,7,27,28$ \\
\hline$\Lambda_{9}$ & 1 & 1 & 1,2 & 1,3 & $1,3,7$ & $1,4,33$ \\
\hline$A_{9}^{(2)}$ & 1 & 1 & 1,2 & $1,2,3$ & 4,6 & $7,22,31$ \\
\hline$K_{10}$ & 1 & 1 & 1,2 & $1,2,3$ & $1,2,3,4,5,6,7$ & $3,7,10,26,27,28,32$ \\
\hline$K_{10}^{\prime}$ & 1 & 1 & 1,2 & 2,3 & $2,4,6$ & $3,7,27,28$ \\
\hline$K_{10}^{\prime *}$ & 1 & 1 & 1 & & & 6,16 \\
\hline$\Lambda_{10}$ & 1 & 1 & 1,2 & 1,3 & $1,3,7$ & $1,4,5,33$ \\
\hline$A_{10}^{(2)}$ & 1 & 1 & 1,2 & 2 & $4,5,6$ & $7,8,10,22,30,31$ \\
\hline$A_{10}^{(3)}$ & 1 & & & & & \\
\hline$A_{10}^{(4)}$ & 1 & 1 & & & & \\
\hline$K_{11}$ & 1 & 1 & 1,2 & $1,2,3$ & $1,2,3,4,5,6,7$ & $3,7,10,26,27,28,32$ \\
\hline$\Lambda_{11}^{\min }$ & 1 & 1 & 1,2 & $1,2,3$ & $1,3,4,6,7$ & $1,4,5,7,8,28,29,31,32,33$ \\
\hline$\Lambda_{11}^{\max }$ & 1 & 1 & 1,2 & 1,3 & $1,3,7$ & $1,4,5,33$ \\
\hline$A_{11}^{(2)}$ & 1 & 1 & 1,2 & 2 & $4,5,6$ & $7,8,10,22,30,31$ \\
\hline$K_{12}$ & 1 & 1 & 1,2 & $1,2,3$ & $1,2,3,4,5,6,7$ & $3,7,10,26,27,28,32$ \\
\hline$\Lambda_{12}^{\min }$ & 1 & 1 & 1,2 & $1,2,3$ & $1,2,3,4,6,7$ & $1,3,4,5,7,8,22,27,28,29,31,32,33$ \\
\hline$\Lambda_{12}^{\mathrm{mid}}$ & 1 & 1 & 1,2 & $1,2,3$ & $1,3,4,6,7$ & $1,4,5,7,8,27,28,29,31,32,33$ \\
\hline$\Lambda_{12}^{\max }$ & 1 & 1 & 1,2 & 1,3 & $1,3,7$ & $1,4,5,33$ \\
\hline$A_{12}^{(2)}$ & 1 & 1 & 1,2 & 2,3 & $4,5,6$ & $7,8,10,22,27,28,29,30,31,32$ \\
\hline$A_{12}^{(3)}$ & 1 & 1 & 1,2 & & & \\
\hline$\Lambda_{13}^{\min }$ & 1 & 1 & 1,2 & $1,2,3$ & $1,2,3,4,6,7$ & $1,3,4,5,7,8,22,27,28,29,31,32,33$ \\
\hline$\Lambda_{13}^{\mathrm{mid}}$ & 1 & 1 & 1,2 & $1,2,3$ & $1,3,4,5,6,7$ & $1,4,5,7,8,10,22,26,27,28,29,30,31,32,33$ \\
\hline
\end{tabular}

TABLEAU 2. Sections parfaites de dimension $\leq 7$ de quelques réseaux de dimension $\geq 7$ (pour chaque réseau $\Lambda$ et chaque $m<\operatorname{dim} \Lambda$ on donne la liste des indices $j$ tels que $P_{m}^{j}$ soit inclus dans $\Lambda$ ). On rappelle que $K_{7}$ et $K_{8}$ ne sont pas parfaits.

Le même tableau inclut aussi trois invariants des réseaux $P_{n}^{j}$ qui ne figurent pas dans [Conway et Sloane 1988b] : l'invariant de Smith, la norme $N^{\prime}$ du réseau dual dans la normalisation qui le rend entier et primitif, et enfin $s^{*}=s\left(\Lambda^{*}\right)=s\left(\Lambda^{\prime}\right)$.

Nous avons utilisé les matrices de Gram obtenues par réduction LLL des matrices de [Conway et Sloane 1988b]. Ces matrices, qui ont l'inconvénient de masquer certaines propriétés, comme l'existence des racines, ont l'avantage de raccourcir les temps d'exécution et de mettre en évidence pour tous les réseaux parfaits de dimension $\leq 7$ des bases formées de vecteurs minimaux.

Nous avons aussi appliqué le programme à certains des réseaux «classiques» $K_{n}, K_{n}^{\prime}, \Lambda_{n}, A_{n}^{(r)}$ de Barnes-Craig (voir l'appendice), de dimensions comprises entre 7 et 13 . Les résultats se trouvent dans le tableau 2. 
Bien que le programme ait été utilisé avec succès dans de nombreux exemples de dimension $\leq 12$, il atteint là parfois la limite de ses possibilités. Pratiquement, sur une station Sparc 3, la mise en évidence d'une inclusion $P_{7}^{i} \rightarrow \Lambda$ prend de quelques secondes à quelques minutes. En revanche, prouver l'impossibilité d'un plongement nécessite parfois des calculs très longs ; par exemple, il a fallu, pour prouver l'impossibilité des plongements dans $K_{12}$ des réseaux $P_{7}^{8}, P_{7}^{22}, P_{7}^{29}$ et $P_{7}^{31}$, environ 53 , 56,30 et 79 heures de temps CPU.

Une question naturelle est de savoir si un réseau donné peut se plonger dans le réseau de Leech. L'examen du tableau 2 permet de répondre à cette question dans le cas des réseaux parfaits de dimension $\leq 7$ :

Théorème 3.7. Tous les réseaux parfaits de dimension $\leq 7$ qui sont entiers pour une norme divisant 4 , et en particulier les réseaux parfaits de dimension $\leq 6$, se plongent dans le réseau de Leech $\Lambda_{24}$.

En effet, on constate sur la table 2 que ces réseaux se plongent tous dans l'un des réseaux $K_{10}$ ou $\Lambda_{12}^{\min }$, à l'exception du réseau $P_{7}^{30}$ pour lequel nous avons eu recours à $\Lambda_{13}^{\text {mid }}$, et que $K_{10}$ contient tous les réseaux parfaits jusqu'à la dimension 6 .

\section{SECTIONS DE PETITE CODIMENSION}

On conserve les notations du paragraphe précédent. En particulier, $\Lambda^{\prime}$ désigne le réseau $\sqrt{q} \Lambda^{*}$, qui est entier et primitif. Si $\mathcal{B}=\left(e_{1}, e_{2}, \ldots, e_{n}\right)$ est une base de $\Lambda$, de matrice de Gram $A$, alors

$$
\mathcal{B}^{\prime}=\left(\sqrt{q} e_{1}^{*}, \sqrt{q} e_{2}^{*}, \ldots, \sqrt{q} e_{n}^{*}\right)
$$

est une base de $\Lambda^{\prime}$, dont la matrice de Gram est $q A^{-1}$; on pose $e_{i}^{\prime}=\sqrt{q} e_{i}^{*}$.

On cherche à explorer les sections parfaites du réseau $\Lambda$ au-delà de la dimension 7 , en commençant par la codimension 1 . Il s'agit toujours de réseaux de même norme que $\Lambda$.

Comme on l'a vu, l'application $M \rightarrow M_{\Lambda^{\prime}}^{\perp}$ met en bijection les sections de $\Lambda$ de dimension $m$ et les sections de $\Lambda^{\prime}$ de dimension $n-m$. En particulier, les sections hyperplanes de $\Lambda$ sont en bijection avec les couples $\pm x$ de vecteurs primitifs de $\Lambda^{\prime}$. Exprimés dans la base $e_{i}^{\prime}$, ce sont les vecteurs dont les composantes sont des entiers premiers entre eux.

Comme norme et déterminant coïncident en dimension 1, le déterminant de la section hyperplane orthogonale à $x$ est donné par la formule

$$
\operatorname{det} M=q^{-1} \operatorname{det} \Lambda N(x) .
$$

On en déduit que les sections hyperplanes les plus denses de $\Lambda$ ont pour déterminant $q^{-1} \operatorname{det} \Lambda N\left(\Lambda^{\prime}\right)$, et que, pour dresser la liste des sections hyperplanes de déterminant majoré par une constante $K$, il suffit d'examiner les vecteurs de $\Lambda^{\prime}$ de norme au plus $q K / \operatorname{det} \Lambda$.

Comme les réseaux parfaits possèdent $n$ vecteurs minimaux indépendants, on peut majorer les déterminants des sections hyperplanes parfaites de $\Lambda$ par $N^{n-1}$, borne déduite de l'inégalité de Hadamard. Dans la pratique, cette majoration est très mauvaise. Dans les dimensions $n \leq 7$, le réseau parfait le moins dense est $A_{n}$, de déterminant $n+1$ et de norme 2, alors que l'inégalité de Hadamard revient à considérer le réseau $\sqrt{2} \mathbb{Z}^{n}$, de déterminant $2^{n}$, remplaçant une majoration linéaire par une majoration exponentielle. Coxeter a conjecturé que $A_{n}$ est le moins dense des réseaux parfaits de dimension $n$. Bien que nous partagions l'opinion émise dans [Conway et Sloane 1998b], à savoir que cette conjecture pourrait bien être fausse dans de grandes dimensions, nous pensons que la densité de $A_{n}$ est une bonne estimation en petites dimensions de la densité minimale des réseaux parfaits de dimension $n$, et nous avons systématiquement limité nos recherches de sections hyperplanes à la borne $K$ correspondant à une section semblable à $A_{n-1}$, qui est $K=n\left(\frac{1}{2} N\right)^{n-1}$.

C'est cette borne que nous avons adoptée pour les dimensions 9 et 10. Au-delà, les calculs deviennent extrêmement longs, et nous ne les avons alors conduits que pour les premières valeurs prises par la norme sur $\Lambda^{\prime}$. 
Par exemple, dans le cas de $\Lambda=K_{12}$, on a $D^{\prime}=$ $3^{6}\left(K_{12}\right.$ est semblable à son dual) et $q=3$, et la borne correspondant à $A_{11}$ est $K=2^{13} 3^{-4} \approx 101$. Nous n'avons examiné que les normes $N^{\prime}=4,6$, $8,10,12,14$ (noter que $\Lambda^{\prime}$ est isométrique à $K_{12}$, donc pair). Le nombre de couples de vecteurs de norme $N^{\prime}$ croît très vite avec $N^{\prime}$ (de 378 pour $N^{\prime}=4$ à 163296 pour $N^{\prime}=14$; voir [Conway et Sloane 1988, tableau 4.11]), ce qui entraîne une croissance rapide du temps de calcul en fonction de la norme. À titre d'exemple, voici les résultats pour $K_{12}$ : il y a à isométrie près une section dans le cas des normes 4, 6, 8, 10,14 et trois dans le cas de la norme 12 , et une unique section parfaite pour chacune des normes $4,6,8,12,14$, la première étant bien entendu $K_{11}$.

Voici quelques indications sur l'algorithme utilisé.

On part d'un réseau $\Lambda$ défini à isométrie près par une matrice de Gram $A$ dont les coefficients sont des entiers premiers entre eux. On calcule d'abord l'invariant de Smith de $\Lambda$ puis la matrice de Gram $A^{\prime}=q A^{-1}$ de $\mathcal{B}^{\prime}$ par une procédure de PARI [Batut et al. 1991]. Les calculs utilisent ensuite des programmes écrits en $\mathrm{C}$ sans multi-précision de façon à limiter les temps de calcul.

On détermine le groupe d'automorphismes de $\Lambda$ (ou de $\Lambda^{*}$, ou de $\Lambda^{\prime}$, c'est la même chose) par une méthode qui nous a été indiquée par Jaquet, puis les orbites de vecteurs de $\Lambda^{\prime}$ jusqu'à une borne calculée en fonction de la borne $K$ choisie pour les déterminants des sections. On se débarasse des vecteurs non primitifs, et l'on retient enfin un vecteur par orbite.

L'étape suivante est la détermination d'une matrice de Gram du réseau $M$, section de $\Lambda$ par l'hyperplan orthogonal à l'un des vecteurs $y \in \Lambda^{\prime}$ retenu. Cela se fait par un algorithme de forme normale d'Hermite (HNF) [Cohen 1993, p. 66] : dans la base $\left(e_{1}^{\prime}, \ldots, e_{n}^{\prime}\right)$, on écrit $y=\sum_{i=1}^{n} h_{i} e_{i}^{\prime}$, les $y_{i}$ étant des entiers premiers entre eux. Par divisions successives par l'un des $y_{i}$ pour lequel $\left|y_{i}\right|$ est minimum parmi les composantes non nulles, on construit un système $\varepsilon_{1}, \ldots, \varepsilon_{n}$ de vecteurs de $\Lambda$ tels que $\varepsilon_{i} \cdot y=0$ pour $1 \leq i \leq n-1$ et $\varepsilon_{n} . y=1$. Alors, $\left(\varepsilon_{1}, \ldots, \varepsilon_{n-1}\right)$ est une $\mathbb{Z}$-base de $M$.

On a ainsi construit un système de représentants des sections hyperplanes de $\Lambda$ de déterminant $\leq K$ modulo le groupe des automorphismes de $\Lambda$. On recherche ensuite les isométries entre sections hyperplanes de façon à avoir finalement un système de représentants modulo isométries des sections hyperplanes de $\Lambda$ de déterminant $\leq K$, et l'on teste la perfection de ces réseaux.

En dimensions $n=9$ et $n=10$, dans le cas des réseaux $\Lambda_{9}, K_{9}, K_{9}^{\prime}, \Lambda_{10}, K_{10}, K_{10}^{\prime}$ et $K_{10}^{\prime *}$, on constate que seul $\Lambda_{9}$ possède une section minimale sembable à $A_{n-1}$, et que les sections hyperplanes parfaites autres que $A_{n-1}$ de ces six réseaux sont de déterminant beaucoup plus petit que celui qu'aurait une section de type $A_{n-1}$, même dans le cas de $K_{9}$ qui contient le moins dense des réseaux de Laïhem. Les réseaux de dimension 8 trouvés en coupant $\Lambda_{9}, K_{9}$ et $K_{9}^{\prime}$ sont tous des réseaux de Laïhem (c'est-à-dire ils possèdent des sections parfaites de même norme en dimension 7). Dans le cas de $\Lambda_{9}$ ce sont les quatre réseaux $E_{8}, A_{8}^{2}, D_{8}, A_{8}$ connus pour posséder l'une des sections $E_{7}, D_{7}$, $A_{7}$; dans le cas de $K_{9}$ ce sont les réseaux $L h_{k}$ pour $k=4,15,16,195,1154,1171$, et dans le cas de $K_{9}^{\prime}$ ce sont les $L h_{k}$ pour $k=3,16,61,1047$.

On indique maintenant comment adapter le procédé précédant à des codimensions $m>1$. C'est possible, pourvu que $m$ soit petit (en pratique 2 ou 3 ).

On doit trouver dans le réseau $\Lambda^{\prime}$ des systèmes de $m$ vecteurs indépendants ayant un déterminant donné soumis aux inégalités imposées par la borne que l'on choisit pour les déterminants des sections. En utilisant la réduction d'Hermite-Korkine-Zolotareff, on exhibe une constante $K_{m}$ telle que tout réseau $M$ de dimension $m$ possède une base $\left(e_{1}, e_{2}\right.$, $\left.\ldots, e_{m}\right)$ qui vérifie les inégalités

$$
N\left(e_{1}\right) N\left(e_{2}\right) \ldots N\left(e_{m}\right) \leq K_{m} \operatorname{det} M
$$

et

$$
N\left(e_{1}\right) \leq N\left(e_{2}\right) \leq \cdots \leq N\left(e_{m}\right)
$$


On peut prendre $K_{m}=\left(\frac{4}{3}\right)^{m(m-1) / 2}$; pour $m \leq 4$, le meilleur choix possible est $K_{m}=\gamma_{m}^{m}$.

On majore ainsi la norme de $e_{1}$, puis celle de $e_{2}$ en fonction de la valeur de $N\left(e_{1}\right), \ldots$, et un calcul fini (mais nécessitant d'importantes mises en mémoire) permet de faire une liste de $m$-uples de vecteurs de $\Lambda^{\prime}$ suffisante pour engendrer toutes les sections de dimension $m$ de $\Lambda^{\prime}$ ayant le déterminant voulu. Il faut ensuite éliminer les $m$-uples de déterminant trop grand et ne retenir qu'un $m$-uple parmi tous ceux qui engendrent la même section. On procède enfin comme dans le cas de la codimension 1 : on retient une section de dimension $m$ de $\Lambda^{\prime}$ par orbite, on calcule les orthogonaux dans $\Lambda$ par un algorithme HNF, on effectue un test d'isométrie, et l'on termine en testant la perfection des réseaux de la liste obtenue.

Nous achevons ce paragraphe avec quelques résultats expérimentaux obtenus par l'application de l'algorithme décrit ci-dessus. On note que, faute de disposer de bonnes minorations de la constante d'Hermite des réseaux parfaits de dimension $\geq 8$, on ne peut pas garantir à partir de la dimension 9 que l'on a trouvé toutes les sections parfaites de même norme d'un réseau donné $\Lambda$. Le tableau 3 donne pour certains réseaux $\Lambda$ de dimension $n=9$ et 10 la liste des sections parfaites de même norme et de codimension 1 ayant un invariant d'Hermite au moins égal à celui de $A_{n-1}$. Il apparaît clairement que la borne de $A_{n-1}$ est très loin d'être atteinte, sauf lorsque le réseau $A_{n-1}$ lui-même est une section, un résultat que l'on observe aussi en dimension 8 sur les tables de Laïhem [1992].

Nous avons vérifié que les sections hyperplanes parfaites de $\Lambda_{9}, K_{9}, K_{9}^{\prime}$ mentionnées dans le tableau 3 sont toutes des réseaux de Laïhem.
Nous avons également comparé les sections de dimension 9 de $K_{10}, K_{10}^{\prime}$ et $K_{10}^{\prime *}$ aux 226 voisins de $D_{9}$ (dont 136 de norme 4 et 27 de norme 6 ), dont la liste a été établie par Jaquet [1992]; on ne trouve que les sections $\mathrm{n}^{\circ} 12$ et $\mathrm{n}^{\circ} 28$ du réseau $K_{10}$.

À partir de la dimension 11, les temps de calcul et les volumes de stockage deviennent prohibitifs, et nous n'avons fait qu'une recherche de sections de petit déterminant, conduisant à 13 sections dans le cas de $K_{11}$, et à seulement 4 dans le cas de $K_{12}$ déjà mentionné (voir la page 4 ).

\section{APPENDICE : QUELQUES RÉSEAUX CLASSIQUES}

Nous donnons maintenant une brève description accompagnée de références des réseaux utilisés dans cet article. Nous donnons aussi pour chaque réseau $\Lambda$ rencontré son invariant Smith, ainsi que, dans le cas des réseaux parfaits de dimension $\leq 7$, la norme $N^{\prime}$ du réseau $\Lambda^{\prime}$ entier et primitif proportionnel à $\Lambda^{*}$ et l'invariant $s^{*}=s\left(\Lambda^{*}\right)$, invariants qui ne se trouvent que rarement dans la littérature (voir les tableaux 1 et 4 ). On déduit de ces invariants la norme du réseau dual et l'invariant $\gamma_{n}^{\prime}(\Lambda)$ de [Bergé et Martinet 1989], à savoir $N\left(\Lambda^{*}\right)=q^{-1} N^{\prime}$ et $\gamma_{n}^{\prime}(\Lambda)=N(\Lambda) N\left(\Lambda^{*}\right)=q^{-1} N N^{\prime}$, où $q$ est le premier terme de $\operatorname{Smith}(\Lambda)$.

Nous ne revenons pas sur les 48 réseaux parfaits de dimension $\leq 7$, pour lesquels nous renvoyons au $\S 2$, à la table 1 , et à l'article [Conway et Sloane 1988b].

Les réseaux de racines irréductibles et primitifs sont $\mathbb{Z}, A_{n}$ pour $n \geq 2, D_{n}$ pour $n \geq 4$, et $E_{n}$ pour $n=6,7,8$ [Coxeter 1951]. $\mathbb{Z}$ et $E_{8}$ sont unimodulaires, et les réseaux $P_{n}^{1}$ pour $1 \leq n \leq 7$ sont $\mathbb{Z}, A_{2}$, $A_{3}, D_{4}, D_{5}, E_{6}, E_{7}$; on a en outre les similitudes

\begin{tabular}{|l|ccccccc|}
\hline réseau & $\Lambda_{9}$ & $K_{9}$ & $K_{9}^{\prime}$ & $\Lambda_{10}$ & $K_{10}$ & $K_{10}^{\prime}$ & $K_{10}^{\prime *}$ \\
\hline nombre de sections & 29 & 57 & 26 & 141 & 189 & 26 & 16 \\
nombre de sections parfaites & 4 & 6 & 5 & 4 & 10 & 3 & 2 \\
numéro des sections & $1,3,6,28$ & $3-6,12,21$ & $1-4,6$ & $1-4$ & $1-5,7,12,14,15,28$ & $1-3$ & 1,2 \\
\hline
\end{tabular}

TABLEAU 3. Sections hyperplanes de réseaux de dimension 9 et 10. On donne successivement le nombre de classes d'isométrie de sections hyperplanes de même norme trouvées, le nombre de ces sections qui sont parfaites et leur numéro dans la liste par invariant d'Hermite décroissant. 
$E_{6}^{*} \sim P_{6}^{2}$ et $E_{7}^{*} \sim P_{7}^{2}$. Les invariants des réseaux $A_{n}$ sont $\operatorname{Smith}\left(A_{n}\right)=n+1, N^{\prime}=n, s^{*}=n+1$. Ceux des réseaux $D_{n}$ sont les suivants: $\operatorname{Smith}\left(D_{n}\right)=2^{2}$ ou 4 selon que $n$ est pair ou impair, $N^{\prime}=2$ ou 4 selon que $n$ est pair ou impair, $s^{*}=12$ ou $n$ selon que $n=4$ ou $n \geq 5$.

Les réseaux laminés $\Lambda_{n}$, pour $0 \leq n \leq 24$, sont décrits dans [Conway et Sloane 1988a, ch. 6]; ils sont entiers de norme 4 . On a $\Lambda_{n} \simeq \sqrt{2} P_{n}^{1}$ pour $1 \leq n \leq 7, \Lambda_{8} \simeq \sqrt{2} E_{8}$, et $\Lambda_{24-n}$ est isométrique à $\Lambda_{n}^{\perp}$ pour $0 \leq n \leq 24$, l'orthogonalité étant calculée dans le réseau de Leech $\Lambda_{24}$. Ils sont uniques à isométrie près sauf dans les dimensions $11,12,13$. Pour $n=11,12$, l'orthogonalité associe $\Lambda_{n}^{\min }$ à $\Lambda_{24-n}^{\min }$ et $\Lambda_{n}^{\max }$ à $\Lambda_{24-n}^{\max }$, pendant que pour $n=12$ elle associe aussi $\Lambda_{n}^{\text {mid }}$ à $\Lambda_{24-n}^{\text {mid }}$.

Pour décrire les réseaux $K_{n}$ (définis pour $0 \leq$ $n \leq 24$ par Leech) et $K_{n}^{\prime}$ (définis dans [Martinet 1992]), on revient à la définition originale de $K_{12}$ [Coxeter et Todd 1953] utilisant des congruences sur l'anneau $A$ des entiers d'Eisenstein. On construit une suite de sections de

$$
\Lambda=K_{12} \supset K_{11} \supset \cdots
$$

de façon que les réseaux obtenus aient la densité la plus grande possible. On constate alors que $K_{10}$ n'est pas stable par $A$. La série $K_{n}^{\prime}$ s'obtient en imposant cette stabilité pour $n=10,8, \ldots$ On peut obtenir les séries $K_{n}$ et $K_{n}^{\prime}$ pour $12 \leq n \leq 24$ en munissant $\Lambda_{24}$ d'une structure sur $A$, en plongeant $K_{12}$ dans $\Lambda_{24}$ de façon compatible avec les structures de $A$ et en se ramenant par orthogonalité aux dimensions $\leq 12$ [Martinet 1992], où les réseaux $K_{n}^{\prime}$ sont identifiés à des réseaux faiblement laminés au-dessus de $K_{12}$ construits par Plesken et Pohst [1993]. (Les réseaux $\Lambda_{n}$, pour $0 \leq n \leq 24$, sont les plus denses connus sauf dans les trois dimensions où ils ne sont pas uniques; de même, les sections et laminations de $K_{12}$ cessent d'être les plus denses à partir des dimensions 10 et 14 où apparaissent les réseaux $K_{n}^{\prime}$ de même densité.)

Comme $\Lambda_{24}$ est unimodulaire, on a les égalités $\operatorname{Smith}(L)=\operatorname{Smith}\left(L^{\perp}\right)$ pour tout réseau entier de norme 4 contenu dans le réseau de Leech, à condition de prendre pour invariant de Smith de $L$ (et de $L^{\perp}$ ) les diviseurs élémentaires de $L^{*} / L$, que $L$ soit ou non primitif. Les réseaux $\Lambda_{n}, K_{n}$ et $K_{n}^{\prime}$ sont parfaits, sauf $K_{3}^{\prime}, K_{4}^{\prime}, K_{7}$ et $K_{8}$. On a $K_{n}^{\prime}=K_{n}$ pour $n=11,12,13$, ainsi que des isométries $K_{n} \simeq \Lambda_{n}$ pour $n \leq 6$ et $n \geq 18, K_{n}^{\prime} \simeq \Lambda_{n}$ pour $n \leq 2$ et $n \geq 22$ [Conway et Sloane 1988a, ch. 6; Martinet 1992]. Avec les notations de [Conway et Sloane 1988b] et de [Barnes 1959], on a aussi

$$
\begin{array}{ll}
K_{8}^{\prime} \simeq A_{8,0} \simeq L_{8}^{4}, & K_{7}^{\prime} \simeq P_{7}^{3}, \\
K_{6}^{\prime} \simeq P_{6}^{2}, & K_{5}^{\prime} \simeq P_{5}^{2} .
\end{array}
$$

Le tableau 4 donne les invariants de Smith de quelques uns de ces réseaux; on trouvera divers compléments dans [Napias 1994].

\begin{tabular}{|cc|cc|cc|cc|}
\hline$\Lambda$ & Smith & $\Lambda$ & Smith & \multicolumn{1}{|c|}{$\Lambda$} & Smith & $\Lambda$ & Smith \\
\hline$K_{7}$ & $24.2^{4}$ & $K_{10}$ & $18.6 .3^{2}$ & $\Lambda_{9}$ & $8.2^{6}$ & $\Lambda_{12}^{\min }$ & $8^{2} .4^{2}$ \\
$K_{8}$ & $12^{2} .2^{2}$ & $K_{10}^{\prime}$ & $6^{2} .3^{3}$ & $\Lambda_{10}$ & $12.4 .2^{4}$ & $\Lambda_{12}^{\operatorname{mid}}$ & $4^{4} .2^{2}$ \\
$K_{8}^{\prime}$ & $9.3^{4}$ & $K_{11}$ & $12.3^{4}$ & $\Lambda_{11}^{\min }$ & $16.4^{2} .2^{2}$ & $\Lambda_{12}^{\max }$ & $4^{2} .2^{6}$ \\
$K_{9}$ & $24.6^{2}$ & $K_{12}$ & $3^{6}$ & $\Lambda_{11}^{\max }$ & $4^{3} .2^{3}$ & $\Lambda_{13}^{\operatorname{mid}}$ & $4^{5}$ \\
$K_{9}^{\prime}$ & $36.3^{3}$ & & & & & & \\
\hline
\end{tabular}

TABLEAU 4. Invariants de Smith (diviseurs élémentaires).

(En considérant des sections hyperplanes successives de densité maximum de l'orthogonal dans $\Lambda_{24}$ de $\Lambda_{13}^{\mathrm{mid}}$, on construit une suite de réseaux de norme 4 dans les dimensions $11,10, \ldots$, qui sont parfaits sauf en dimension 8 et qui coïncident avec les $\Lambda_{n}$ pour $n \leq 7$; cette coïncidence en dimension 7 rend compte de la possibilité découverte par Plesken et Pohst [1993] de plonger $\Lambda_{13}^{\text {mid }}$ dans $\Lambda_{17}$.)

Nous avons aussi étudié les réseaux de différences de Craig $A_{n}^{(r)}$ [Conway et Sloane 1988a, ch. 8, §6], qui sont parfaits de minimum $2 r$ pour $r=2$ (ce sont les réseaux $P_{n}$ de [Barnes 1957]) et conjecturalement lorsque $n+1$ est premier et que $r$ est assez petit par rapport à $n$ [Bachoc et Batut 1992, $\S 3]$. Ces réseaux fournissent parfois d'intéressants exemples de réseaux parfaits possédant peu de sections parfaites de même norme. 


\section{BIBLIOGRAPHIE}

[Bachoc et Batut 1992] Ch. Bachoc et Ch. Batut, "Étude algorithmique de réseaux construits avec la forme trace", Experimental Mathematics 1 (1992), 183-190.

[Batut et al. 1992] C. Batut, D. Bernardi, H. Cohen and M. Olivier, User's Guide to PARI-GP, 1992. Ce manuel fait partie de la distribution du programme, disponible sur le serveur megrez.ceremab.u-bordeaux.fr.

[Barnes 1957] E. S. Barnes, "The perfect and extreme senary forms", Canad. J. Math. 9 (1957), 235-242.

[Barnes 1959] E. S. Barnes, "The construction of perfect and extreme forms I, II", Acta Arith. 5 (1959), 57-79 et $461-506$.

[Bergé et Martinet 1989] A-M. Bergé et J. Martinet, "Sur un problème de dualité lié aux sphères en géométrie des nombres", J. Number Theory 32 (1989), 14-42.

[Cohen 1993] H. Cohen, A Course in Computational Algebraic Number Theory, Graduate Texts in Math. 290 (1993), Springer-Verlag.

[Conway and Sloane 1988a] J. H. Conway et N. J. A. Sloane, Sphere Packings, Lattices and Groups, Grundlehren der Math. Wissenschaften 290 (1988), Springer-Verlag.

[Conway and Sloane 1988b] J. H. Conway et N. J. A. Sloane, "Low-dimensional lattices III: Perfect forms", Proc. Royal Soc. London A418 (1988), 4380 .
[Coxeter 1951] H. S. M. Coxeter, "Extreme forms", Canad. J. Math. 3 (1951), 391-441.

[Coxeter and Todd 1953] H. S. M. Coxeter and J. A. Todd, "An extreme duodenary form", Canad. J. Math. 5 (1953), 384-392.

[Jaquet 1992] D-O. Jaquet, "Description des voisines de $E_{7}, D_{7}, D_{8}$ et $D_{9}$ ", Séminaire de Théorie des Nombres de Bordeaux 4 (1992), 273-374.

[Jaquet 1993] D-O. Jaquet, "Énumération complète des classes de formes parfaites en dimension 7", Ann. Inst. Fourier 43:1 (1993), 21-55.

[Korkine et Zolotareff 1877] A. Korkine et G. Zolotareff, "Sur les formes quadratiques positives", Math. Ann. 11 (1877), 242-292.

[Laïhem 1992] M. Laïhem, "Construction algorithmique de réseaux parfaits", Thèse Univ. Bordeaux (1992).

[Martinet 1992] J. Martinet, "Structures algébriques sur les réseaux", Séminaire de Théorie des Nombres de Paris 1992-93, Cambridge Univ. Press (à paraître).

[Napias 1994] H. Napias, "Sur quelques réseaux contenus dans les réseaux de Leech et de Quebbemann", Comptes Rendus Acad. Sc. Paris, à paraître.

[Plesken et Pohst 1993] W. Plesken et M. Pohst, "Constructing integral lattices with prescribed minimum II" Math. Comp. 60 (1993), 817-825.

[Voronoï 1908] G. Voronoï, "Nouvelles applications des paramètres continus à la théorie des formes quadratiques I: Sur quelques propriétés des formes quadratiques positives parfaites", J. reine angew. Math. 133 (1908), 97-178.

Christian Batut, Mathématiques, Université Bordeaux 1, 351, cours de la Libération, F-33405 Talence cedex, France (batut@ceremab.u-bordeaux.fr)

Jacques Martinet, Mathématiques, Université Bordeaux 1, 351, cours de la Libération, F-33405 Talence cedex, France (martinet@ceremab.u-bordeaux.fr)

Received February 22, 1994; accepted May 20 